\title{
Single-Incision Robotic Surgery
}

\author{
Norihiko Ishikawa", Masahiko Kawaguchi, Hideki Moriyama, Go Watanabe \\ Department of General and Cardiothoracic Surgery, Kanazawa University, Kanazawa, Japan \\ Email: \#iskwnrhk@gmail.com
}

Received October 28, 2011; revised December 29, 2011; accepted January 5, 2012

\begin{abstract}
Introduction: Single Incision Laparoscopic Surgery (SILS ${ }^{\mathrm{TM}}$ ) has been developed as a less invasive laparoscopic surgery. On the other hand, robotically assisted surgical technology has offered new options for minimally invasive surgery. In this study, we report a new surgical technology to perform SILS using the da Vinci S surgical system (Intuitive Surgical Inc., Sunnyvale, CA). Materials and Surgical Technique: A porcine liver with gallbladder was placed on an endoscopic surgery trainer, and a $25 \mathrm{~mm}$ incision was made for one robotic camera and two instruments at the umbilicus position. Both instruments were crossed while preventing them from colliding with each other, and Robot-assisted single-incision cholecyctectomy was perfumed. Discussion: This technique is expected to contribute to the development of a number of procedures in the future.
\end{abstract}

Keywords: Single-Incision Laparoscopic Surgery; Robotic Surgery; Surgical System of da Vinci S

\section{Introduction}

Single Incision Laparoscopic Surgery (SILS ${ }^{\mathrm{TM}}$ ) has been developed as a surgical procedure that is less invasive than conventional laparoscopic surgery. The first scientific paper on SILS, in which laparoscopic appendectomy and cholecyctectomy were reported, was published in 1998 $[1,2]$. A problem with SILS is the non-intuitive association between the right and left laparoscopic instruments and the surgeon's hands on the monitor when performing dissecting and ligation in the abdominal/thoracic cavity. At the same time, robotically assisted surgical technology has offered new options for minimally invasive surgery, as evidenced by the large number of papers published on procedures since the first case of complete laparoscopic cholecystectomy was reported in 1998 [3]. In this study, we shall introduce a new surgical technology to perform SILS using the da Vinci S surgical system (Intuitive Surgical Inc., Sunnyvale, CA).

\section{Materials and Surgical Technique}

A porcine liver with gallbladder was placed on a Tuebingen MIS Trainer (Richard Wolf GmbH Co., Knittlingen, Germany) (Figure 1(a)) [4]. A $25 \mathrm{~mm}$ incision was made for one robotic camera and two Endowrist ${ }^{\circledR}$ instruments (Intuitive Surgical Inc.) at the umbilicus position, and a Wound Retractor (Applied Medical, Rancho Santa Margarita, CA) was placed transumbilically and covered

\footnotetext{
*Drs. Norihiko Ishikawa, Masahiko Kawaguchi, Go Watanabe have no conflicts of interest or financial ties to disclose.

"Corresponding author.
}

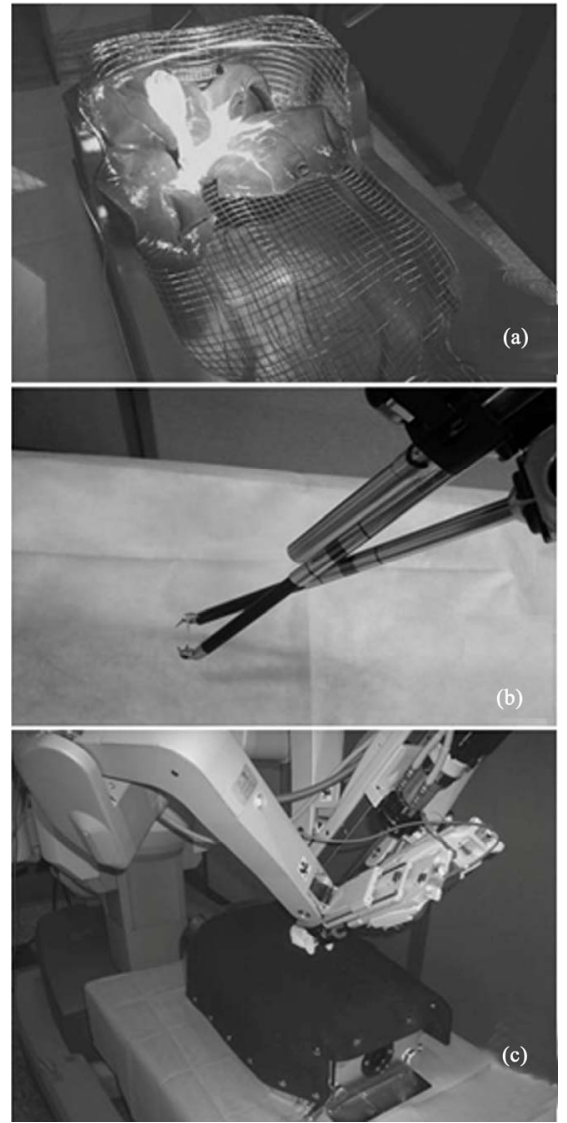

Figure 1. (a) Tuebingen MIS Trainer and the porcine liver with gallbladder; (b), (c) Trocar positioned for Single-incision Robotic Surgery (b) without and (c) with Tuebingen MIS Trainer. 
with a surgical glove to avoid $\mathrm{CO}_{2}$ leakage. A camera trocar $(12 \mathrm{~mm})$ and two instrument cannulaes $(8 \mathrm{~mm})$ were inserted through the fingers of the glove before they were connected to each robotic instrument arm. The da Vinci Surgical System was positioned at the head of the trainer (Figures 1(b) and 1(c)). We also performed this procedure through the pseudoabdominal wall of this trainer (metallic wide mesh and a cover made of neoprene). Both instruments were inserted through the umbilical incision and crossed while preventing them from colliding with each other (Figure 2(a)). At the same time, the master-instrument association at the surgeon console was set to the reverse of default settings so that the right master would control the left instrument and the left master would control the right instrument (Figure 2(b)). We performed robot-assisted cholecyctectomy with the 30 degree up robotic camera and EndoWrist ${ }^{\circledR}$ instrumentsHot Shears and Maryland Bipolar Forceps attached to the surgical arms as in standard laparoscopic cholecyctectomy (Figure 2(c)).

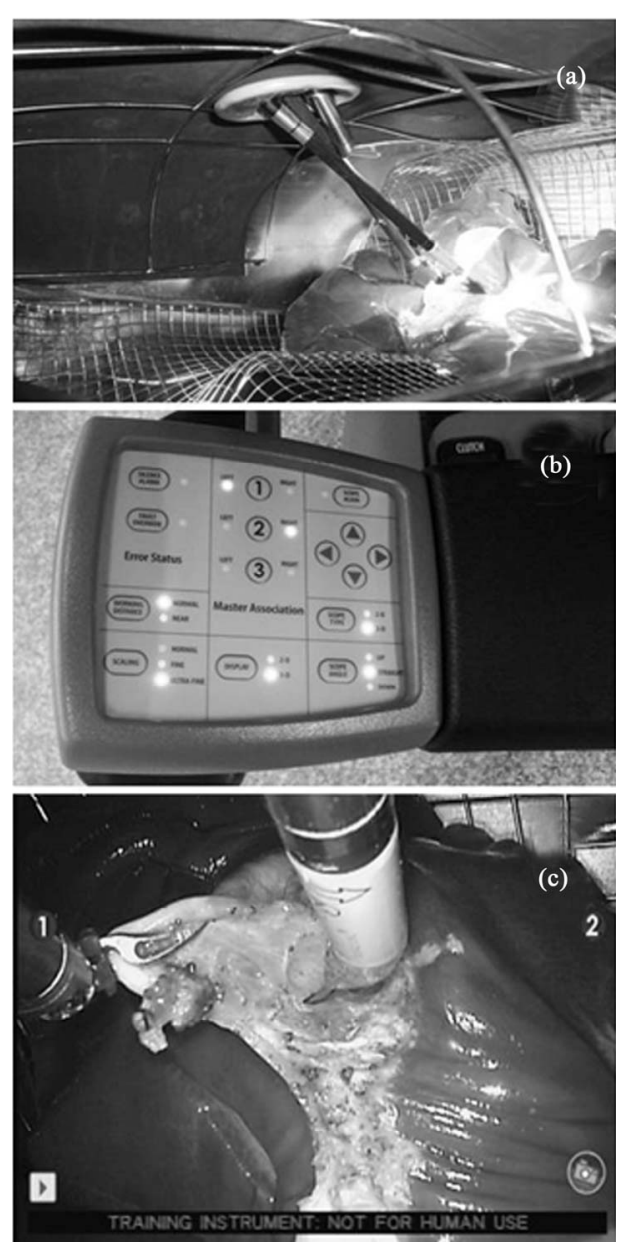

Figure 2. (a) Operative view from inside the Tuebingen MIS Trainer in single-incision robotic cholecystectomy; (b) User Interface Panel at the surgeon console; (c) Dissection of the gallbladder using robotic instruments.
With the porcine laparoscopic cholecystectomy model placed on the Tuebingen MIS Trainer, we performed single-incision robotic surgery. Both cystic duct and cystic artery were dissected, ligated with 3-0 Vicryl suture (Ethicon, Inc. Somerville, NJ) using EndoWrist Maryland Bipolar Forceps and EndoWrist DeBakey Forceps. EndoWrist Hot Shears was used for cutting the cystic duct and artery. No instrument collision occurred during the dissection of the gallbladder. The elapsed time for this procedure was 35 minutes.

\section{Discussion}

In SILS, roticulator instruments were used to create necessary operative angles because their insertion points were much closer to each other. The da Vinci surgical system provides robotic instruments with 7 degrees of freedom -1 degree (inner yaw of the wrist) added to conventional laparoscopic instruments. Because of this, the robotic instruments have more flexible movements than roticulator instruments do. As a result, the surgeon can manipulate the instruments without causing collision even if the instruments are inserted through the same incision. Although we used $8 \mathrm{~mm}$ EndoWrist ${ }^{\circledR}$ instruments in this experiment, use of $5 \mathrm{~mm}$ EndoWrist ${ }^{\circledR}$ instruments would make the surgical technique much easier because of their flexible snake profile (Figure 3). In general, roticulator forceps are used to avoid instrument collision; however, they are not suitable for suturing because their unique jaw mechanism is unable to rotate while bending. The robotic instruments have more degrees of freedom, which enable the surgeon to perform more precise and accurate manipulation. In this study, the cystic duct and cystic artery were ligated and resected with the robotic instruments. This is the one of the virtue of robotic maneuver, and so surgical clip did not need.

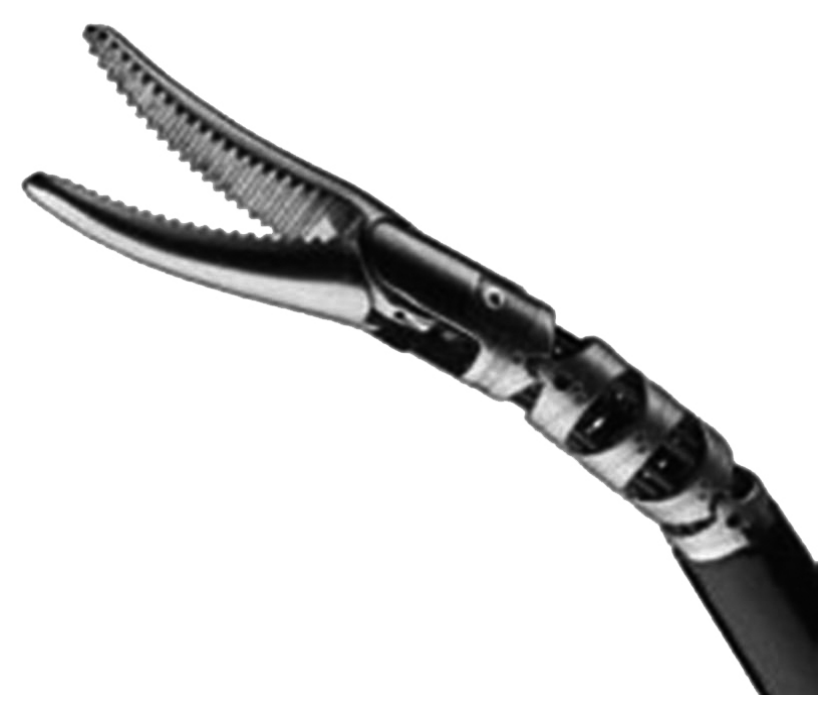

Figure $3.5 \mathrm{~mm}$ EndoWrist ${ }^{\circledR}$ instruments. 
In conventional SILS, the right and left instruments are different from what appears on the monitor. Because of this, the surgeon is normally forced to manipulate the instruments using reverse hands. SILS with roticulator instruments can be more challenging in terms of difficulty due to unstable forced reverse-hand manipulation in addition to the limited movement and accuracy of the instruments. On the other hand, the da Vinci surgical system offers computer-controlled manipulation of robotic instruments, which enables the surgeon to avoid forced reverse-hand manipulation by changing the settings of the master-instrument association at the surgeon console. Thanks to this unique function, the surgeon can manipulate the instruments intuitively by viewing what appears on the monitor without feeling the gap between the right and the left. In addition to this, other advantages of the da Vinci surgical system such as $3 \mathrm{D}$ visualization and accurate micromanipulation can be made use of at the same time. Besides, selection of camera type is very important. The outside arm of 0 degree straight camera is easy to collide with both instrument arms outside of the abdominal cavity, and 30 degree up or 30 degree down camera must be used.

The da Vinci S surgical system provides master association function of which computer program enables a surgeon to swap his left and right hands, EndoWrist instruments with 7 degrees of freedom and high definition $3 \mathrm{D}$ vision. Thanks to these unique functions, intuitive single incision endoscope surgery can be performed with increased safety and speed. The problem is a cost issue, however, additional cost compared with conventional SILS is the cost for instruments and drapes. Having said that, considering that SILS usually uses disposable instruments and that robotic surgery can be performed without clips, the cost may not be a big issue.

This study demonstrated that this computer-controlled robotic characteristic allows for a robot-assisted laparoscopic surgery with only one incision instead of three ports. In addition, further enhanced minimally invasive surgery will be possible with the combination of the SILS concept and the robot-assisted surgery for less invasive laparoscopic surgery. We could show the effectiveness of single incision endoscopic surgery, which can be expected to expand its application area from simple procedure such as colecystectomy to more complicated procedures such as colectomy, etc. This technique is expected to contribute to the development of a number of procedures in the future.

\section{REFERENCES}

[1] C. Esposito, "One-Trocar Appendectomy in Pediatric Surgery,” Surgical Endoscopy, Vol. 12, No. 2, 1998, pp. 177-178. doi:10.1007/s004649900624

[2] G. Piskun and S. Rajpal, “Transumbilical Laparoscopic Cholecystectomy Utilizes No Incisions Outside the Umbilicus," Journal of Laparoendoscopic \& Advanced Surgical Techniques A, Vol. 9, No. 4, 1999, pp. 361-364. doi:10.1089/lap.1999.9.361

[3] J. Himpens, G. Leman and G. B. Cadiere, "Telesurgical Laparoscopic Cholecystectomy,” Surgical Endoscopy, Vol. 12, No. 8, 1998, p. 1091. doi:10.1007/s004649900788

[4] M. Waseda, N. Inaki, L. Mailaender and G. F. Buess, “An Innovative Trainer for Surgical Procedures Using Animal Organs," Minimally Invasive Therapy \& Allied Technologies, Vol. 14, No. 4, 2005, pp. 262-266. doi:10.1080/13645700500273841 